\title{
Auge económico y conflictividad laboral en las telecomunicaciones argentinas: un análisis de los determinantes de la protesta laboral en el sector durante la posconvertibilidad*
}

Economic growth and labor dispute in the argentine telecommunications. An analysis of the determinants of labor protest in the sector during the postconvertibility period

\author{
Martín Rodríguez Miglio**
}

Resumen: En el presente trabajo abordamos la relación entre la conflictividad laboral y el desempeño económico del sector telecomunicaciones durante etapa de la posconvertibilidad en argentina. Así, observamos que en este sector de actividad, la conflictividad presenta dos subperíodos bien diferenciados, tanto en términos cuantitativos como cualitativos. En el primero, 2006-2010, el conflicto es de menor intensidad y motivado principalmente por causas salariales, mientras que entre $2011 \mathrm{y}$ 2015, el conflicto se intensifica, se radicalizan las medidas de protesta y se modifican sus causas, ganando protagonismo las disputas por condiciones de trabajo, principalmente los conflictos por tercerización.

Palabras clave: Conflicto laboral, Tercerización, Telecomunicaciones, Posconvertibilidad

\begin{abstract}
In this paper we address the relationship between labor unrest and economic performance of the telecommunications sector during the post-convertibility period in Argentina. Thus we see that in this sector, the conflict has two distinct sub-periods, both in quantitative and qualitative terms. In the first, 2006-2010, the conflict is less intense and mainly driven by wage causes, while between 2010 and 2015, the conflict intensifies, the protests radicalized and their causes are changed, gaining prominence disputes by working conditions, particularly conflicts outsourcing.
\end{abstract}

Keywords: Labor conflict, Outsourcing, Telecommunications, Postconvertibility

Recibido: 15 abril 2016

Aceptado: 14 agosto 2016

\footnotetext{
* Una versión preliminar ha sido presentada en las VIII Jornadas de Jóvenes Investigadores del Instituto de Investigaciones Gino Germani, Buenos Aires, en noviembre de 2015

** Argentino. Licenciado en Economía por la Facultad de Ciencias Económicas (FCE-UBA), y Doctorando en Ciencias Sociales (UNGS-IDES). Investigador-Docente asistente del Área Economía del Conocimiento, Instituto de Industria, Universidad Nacional de General Sarmiento (IDEI-UNGS). Contacto: mrmiglio@ungs.edu.ar
} 


\section{Introducción}

Durante la década de los noventa la economía argentina estuvo sometida a la vigencia de un régimen de convertibilidad cambiaria que establecía la paridad entre la moneda local y el dólar estadounidense (1 a 1). Este régimen de tipo de cambio apreciado se sostuvo a base del ingreso de divisas por privatizaciones y endeudamiento, pero hacia finales de la década la imposibilidad de conseguir las divisas necesarias que sostuvieran dicho régimen impuso la necesidad de una devaluación y la consecuente derogación de la ley de convertibilidad. La fase de crecimiento económico surgida luego de la devaluación de 2002 en argentina impactó modificando la relación de fuerzas entre capital y trabajo, y eso se evidenció entre otras cosas, en la intensificación de la actividad sindical.

Distintos autores afirman que el nuevo escenario surgido a partir de la devaluación, denominado posconvertibilidad, habría permitido un fortalecimiento de la acción sindical mediante la profundización de las instancias de negociación, el crecimiento en los niveles de afiliación y el traslado del conflicto social a la esfera laboral (Senen González et al, 2010; Trajtemberg, 2011; Barattini, 2013). El primero de los aspectos refiere a la negociación colectiva tanto en una dimensión intensiva (cantidad de trabajadores comprendidos) como extensiva (cantidad de negociaciones). En cuanto a la revitalización de la instancia negocial, esta etapa contrasta significativamente con los niveles de los años previos a la devaluación donde escasamente superaban los 200 acuerdos/convenios al año, mientras que a partir de 2003 comienza una tendencia creciente hasta alcanzar su máximo en 2010 con poco más de 2000 negociaciones. En el presente trabajo nos ocuparemos del tercer elemento, la conflictividad laboral, y su relación con el desempeño económico, intentando identificar una posible explicación sobre las causas que desató el ciclo de protesta ascendente de los últimos años.

En este trabajo intentaremos identificar los determinantes de la protesta laboral en un sector de actividad particular, el de las telecomunicaciones, y analizarlo en relación con el ciclo económico ascendente en el que se encuentra desde la salida de la convertibilidad. Este ciclo se encuentra motorizado principalmente por la emergencia de nuevos servicios de telecomunicaciones que se originaron a partir de la masificación de las nuevas tecnologías. Luego de esta introducción, avanzaremos con el desarrollo del trabajo partiendo de una breve caracterización de la conflictividad general del período, para luego dar paso al análisis sectorial, primero en su dimensión del desempeño económico y luego en cuanto a la conflictividad laboral. Seguidamente analizaremos los conflictos por tercerización, a la luz de haberlos identificado como uno de las fuentes principales de conflicto. Habiendo desarrollado el caso particular, nos detendremos en recapitular el debate sobre la relación entre ciclo y conflicto y su vigencia ante la protesta actual. Finalmente presentaremos algunas conclusiones y reflexiones sobre la relación entre ciclo económico, protesta laboral y condiciones de trabajo en el sector, que entendemos deberán ser retomadas en futuros trabajos. 


\section{Protesta laboral y desempeño reciente del sector telecomunicaciones}

\section{La conflictividad general de un período de crecimiento}

Nos interesa analizar lo sucedido en los años recientes en argentina, a partir de la salida del régimen de convertibilidad, hacia fines del año 2001, y la consecuente devaluación del peso en enero del año siguiente. La nueva estructura de precios relativos permitió la recuperación del nivel de actividad que se fundó principalmente en dos elementos: la fuerte reducción del costo salarial que implicó la devaluación vía caída del salario real, y la recuperación de la rentabilidad de los capitales locales dedicados al mercado interno, producto del encarecimiento de los bienes extranjeros. A este escenario debe sumarse la coyuntura internacional favorable que beneficio a los exportadores agrarios con altos precios de los comodities. Así es como se configura un proceso de considerable expansión económica a nivel local.

Como hemos mencionado anteriormente, la posconvertibilidad también se caracteriza por ser un período donde la actividad sindical sufre una suerte de revitalización en varias de sus formas, pero particularmente en términos de conflictividad laboral. Pues bien, a partir de la salida de la crisis de 2001/2002 en la Argentina nos encontramos con un escenario en el que el ciclo económico ascendente fue acompañado de una conflictividad laboral creciente hasta el año 2010, de manera correlacionada. El año 2011 presentó una tasa de crecimiento económico positiva con un nivel de conflicto estable, para luego pasar a un año de estancamiento del producto con conflictividad creciente, tendencia que se mantuvo en 2014. La serie de conflictos laborales (gráfico 1) se ha construido a partir de la información del Ministerio de Trabajo argentino que cuenta con tal información a partir del año 2006.

Gráfico 1. Conflictividad laboral y crecimiento económico. Año base 2006=100

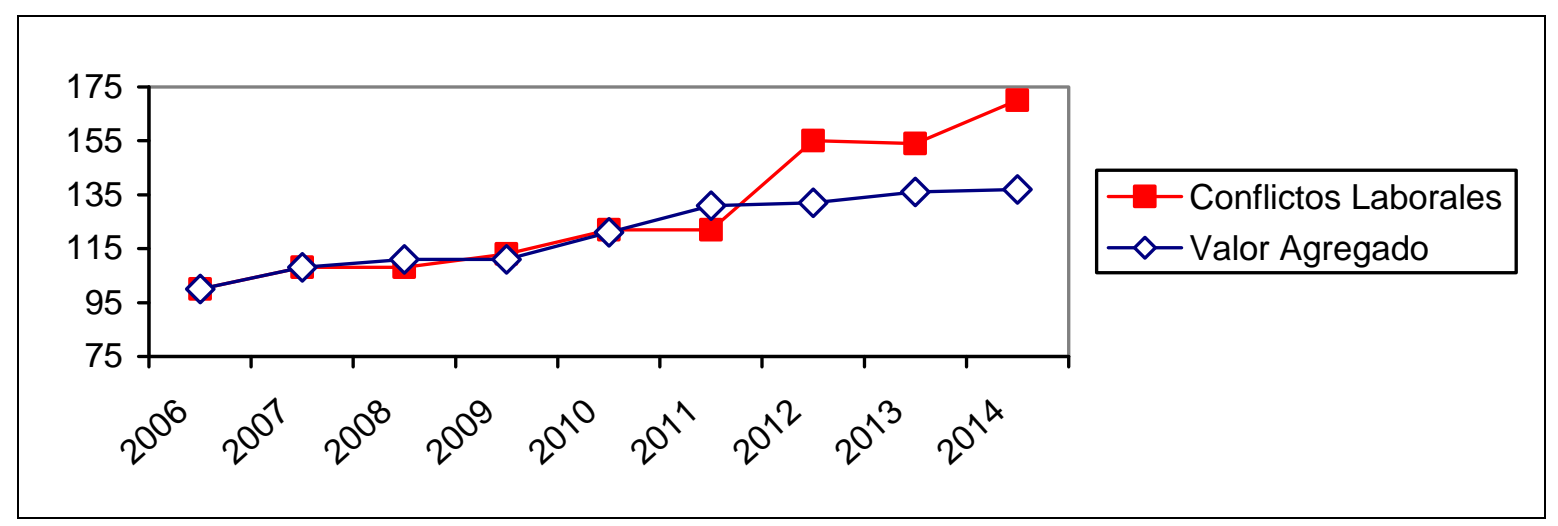

Fuente: Elaboración propia en base a MTSS e INDEC 
Al respecto, diversos autores enfatizan en distintos determinantes de este ciclo de ascenso de la conflictividad, anclados en las dimensiones política, económica o de organización colectiva. Filipetto et al (2015) brindan una interpretación de la intensificación de los conflictos basada tanto en la dimensión económica (puja distributiva, presiones inflacionarias y cambiarias) como políticas (fragmentación de las centrales sindicales). Sin embargo existen otros trabajos donde se enfatiza la dimensión organizativa de los colectivos de trabajadores. Así, Spaltenberg (2012) rechaza la tesis que postula el incremento de la conflictividad del periodo fundado en tensiones extrasindicales o actores intersindicales, y plantea una hipótesis donde el aumento de los conflictos podría deberse a cambios en el actor sindical. Por su parte, Lenguita (2011) enfatiza que la revitalización sindical tiene como uno de sus ejes fundamentales la recuperación de las luchas en los lugares de trabajo.

A través del sector de las telecomunicaciones realizaremos un acercamiento tanto al desempeño económico como al ciclo de protesta para pensar la relación entre ambas. La razón por la que hemos escogido este sector de actividad y no otro radica en el desempeño económico exhibido a lo largo del período. En el sector de las telecomunicaciones el ciclo económico se ha mantenido en alza durante todo el período incluso en los años de bajo o nulo crecimiento económico general ${ }^{1}$. No así la conflictividad, ya que ha experimentado oscilaciones en su intensidad. Profundizaremos entonces en las causas de la conflictividad mediante un análisis concreto de manera de mejorar nuestra caracterización de la relación entre ciclo económico y protesta laboral, o de hallar nuevas variables determinantes en este fenómeno.

\section{El desempeño económico de las telecomunicaciones}

El sector de las telecomunicaciones presenta un alto crecimiento sostenido en la etapa de la posconvertibilidad que ha implicado un incremento en ventas cercano al $400 \%$ medido a pesos constantes de 2008. Como se observa en el cuadro 1, las ventas anuales para el 2014 se ubican casi cuatro veces por encima de las computadas para el año 2003. Si bien corresponde aclarar que los niveles de ventas el 2003 son inferiores a los de la década previa (cerca de un 50\% por debajo de las ventas de los `90), ya en el año 2006 el volumen de ventas a precios constantes supera los valores inmediatos previos a la crisis.

Una buena parte de la explicación de esta evolución debe rastrearse en la expansión de los nuevos servicios surgidos al calor de la revolución tecnológica que implicó la era digital. Así observamos que en el mercado de las telecomunicaciones se incorporaron nuevos servicios que han motorizado el crecimiento del sector, fundamentalmente la telefonía móvil y los servicios de internet (Rodríguez Miglio, 2016).

Por el lado del empleo, la salida de la crisis también ha implicado que en el sector se incorporaran trabajadores registrados, sin embargo dicha incorporación se ha realizado a

\footnotetext{
${ }^{1}$ Nos referimos a la evolución del valor agregado para la descripción del Clanae nro. 642/3/4 - Servicios de transmisión de radio y televisión, telecomunicaciones e internet.
} 
una tasa significativamente menor que la del crecimiento de las ventas. El incremento de los puestos de trabajo además no ha sido equilibrado, dado que la mayoría de los mismos corresponde a los primeros años de la posconvertibilidad, entre 2003 y 2008. A partir de allí la incorporación de trabajadores registrados en el sector gravitó en torno a los 78 mil puestos, con pequeñas variaciones año a año, tanto positivas como negativas.

Cuadro 1. Desempeño de las telecomunicaciones y situaciones de conflicto, 2003-2015

\begin{tabular}{l|lccc|}
\multicolumn{1}{l}{ Año } & Ventas* & $\begin{array}{c}\text { Tasa de } \\
\text { crecimiento de las } \\
\text { ventas }\end{array}$ & $\begin{array}{l}\text { Empleo } \\
\text { registrado }\end{array}$ & $\begin{array}{l}\text { Conflictos } \\
\text { laborales }\end{array}$ \\
\hline 2003 & 13.045 & $13,53 \%$ & 58.316 & s/d \\
2004 & 18.073 & $38,54 \%$ & 61.267 & s/d \\
2005 & 19.293 & $6,75 \%$ & 65.415 & s/d \\
2006 & 21.243 & $11,04 \%$ & 69.978 & 11 \\
2007 & 24.873 & $16,10 \%$ & 74.285 & 6 \\
2008 & 28.541 & $14,75 \%$ & 77.958 & 0 \\
2009 & 29.546 & $3,52 \%$ & 77.402 & 2 \\
2010 & 31.545 & $6,77 \%$ & 77.528 & 5 \\
2011 & 38.524 & $22,12 \%$ & 76.132 & 12 \\
2012 & 45.284 & $17,55 \%$ & 76.862 & 6 \\
2013 & 49.731 & $9,82 \%$ & 77.581 & 6 \\
2014 & 50.352 & $1,25 \%$ & 78.379 & 5 \\
2015 & s/d & $2,80 \% * *$ & s/d & 20 \\
$*$ en millones de pesos deflactados según IPC-Indec, Año base=2008 & \\
$* *$ estimación de la Cámara Argentina de la Construcción (2015). &
\end{tabular}

Fuente: Elaboración propia en base a CICOMRA y ODS-CTA

En relación al conflicto, el sector presenta años de diversa intensidad. Los años 2006, 2011 y 2015 manifiestan la mayor concentración de conflictos laborales (11, 12 y 20 respectivamente), mientras que 2008 y 2009 son años de relativa calma con mínima o nula presencia de conflictos. El resto de los años la conflictividad se acerca al promedio de la serie. En función de los datos observados en el cuadro 1, y teniendo en consideración los visto previamente en relación a la conflictividad general, podemos observar que el comportamiento combativo de los trabajadores de las telecomunicaciones pareciera estar por debajo del promedio general, aun siendo un sector de actividad que transita por una clara fase de crecimiento, lo que podría pensarse como la posibilidad de disputar una masa de excedente que se encuentra en crecimiento. Surgen entonces algunos interrogantes relacionados con los móviles más importantes de este ciclo de protesta entre los trabajadores de las telecomunicaciones, así como también sobre la forma que adopta y los actores intervinientes. Ante este escenario cobra relevancia la necesidad de un análisis más profundo de la protesta, tanto cuantitativo como cualitativo, que permita entender su relación o no con el ciclo económico en expansión y sus determinantes fundamentales. 


\section{La protesta laboral en el sector}

En argentina la actividad sindical del sector de las telecomunicaciones se encuentra fuertemente fragmentada, principalmente en lo que se refiere al personal de base. Dicha fragmentación se arrastra desde la etapa de la convertibilidad, la década del noventa, momento en que la Foetra Bs. As. decidió su desafiliación de la federación en la que se encontraba nucleada, la FOESSITRA. Esta desafiliación fue acompañada por los sindicatos telefónicos de Chaco, Tucumán, Santa Fe, Rosario y Luján, quienes posteriormente conformarían una nueva federación denominada FATEL (Senén González y Garro, 2007).

En la actualidad la FOESSITRA se encuentra constituida por 24 sindicatos (Bahía Blanca, Soeesit Bs. As., Catamarca, Córdoba, Corrientes, Chaco, Chubut, Entre Ríos, Formosa, Jujuy, La Pampa, La Plata, La Rioja, Mar del Plata, Mendoza, Misiones, 9 de Julio, Pergamino, Río Cuarto, Salta, San Juan, Santiago del Estero, Tandil y San Luis), y la FATEL por 6 (Foetra Bs. As., Soeesit Santa Fe, Suttach Chaco, Soettuc Tucumán, Sitratel Rosario y Siloe\&esit Luján). Asimismo se encuentran otras tres organizaciones sindicales que nuclean a técnicos, profesionales y jerárquicos telefónicos. Nos referimos a la Federación de Personal de Supervisión y Técnico (FOPSTTA), a la Unión de Personal Jerárquico (Upjet) y al Centro de Profesionales Universitarios de las empresas de Telecomunicaciones (Cepetel). En los últimos años ha surgido un nuevo sindicato, el Uettel, con intenciones de representar a los trabajadores telefónicos tercerizados que mayormente se encontraban encuadrados en el convenio para obras de telefonía de la Uocra. El sindicato que detenta la mayor representación de los trabajadores en términos de afiliados es el Foetra Bs. As. (Aruguete y Duarte, 2005)

A pesar de dicha fragmentación en diversos colectivos de trabajadores, el sector presenta un nivel de conflictividad que resulta visible en el marco de la protesta laboral general, al menos en algunos años en particular y debido quizás a la radicalidad de las medidas adoptadas ${ }^{2}$. Como vimos anteriormente, entre el año 2006 y 2015 se identificaron 73 situaciones de conflicto en el sector de las telecomunicaciones argentinas, alcanzando un pico de conflicto hacia el último de los años, con 20 casos, mientras que también se destacan los años 2006 y 2011, con 11 y 12 conflictos respectivamente. De la serie desplegada en el gráfico 2 se observa que el nivel de conflictividad en estos diez años ha sido oscilante, con un promedio que supera levemente los 7 conflictos por año, pero reflejando en ocasiones años de baja conflictividad, como los años 2008 y 2009.

\footnotetext{
${ }^{2}$ Podemos mencionar por ejemplo los acampes realizados por el Uettel en la puerta del edificio de Telefónica de Argentina en pleno microcentro porteño, sostenido durante varios días, en 2013 y 2015.
} 
Gráfico 2. Conflictividad laboral en las telecomunicaciones, 2006-2015

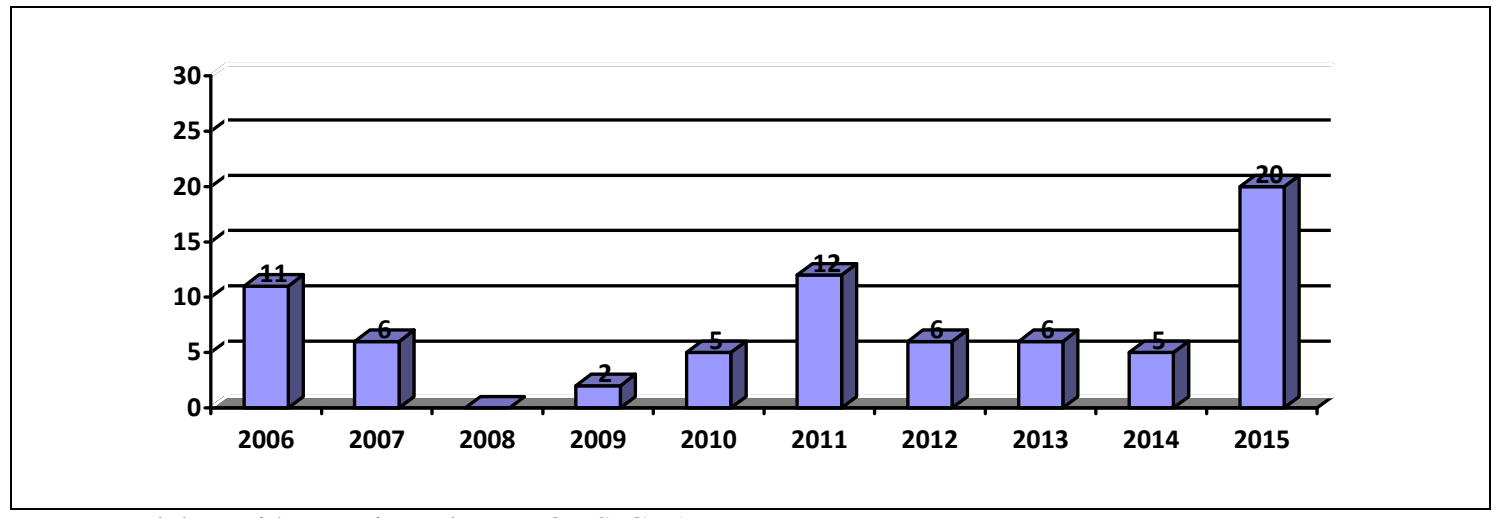

Fuente: Elaboración propia en base a ODS-CTA

En estos 73 casos de conflicto observados se destaca la presencia de tres asociaciones sindicales intervinientes: el Uettel (Unión de Empleados Técnicos de las Telecomunicaciones) en 19 conflictos del panel (26\%), el Cepetel (Sindicato de los Trabajadores de las Tecnologías de la Información y la Comunicación) en 14 (19\%), y la Foetra Bs. As. (Sindicato de las Telecomunicaciones) en 11 de los conflictos (15\%). En menor medida surgen como actores del conflicto una dispersión de sindicatos regionales o agrupaciones tales como Atic, Soetc, Sindicato de Telefónicos de Corrientes, etc. (32\% sumados todos juntos). Cabe destacar la importante presencia de conflictos asumidos por trabajadores sin representación gremial a lo largo de estos años en un $8 \%$ de los casos.

A nivel empresa podemos destacar que los conflictos laborales del sector se encuentran fuertemente orientados hacia las dos compañías telefónicas con mayor presencia en la rama: Telecom (23\% de los casos) y Telefónica (32\%) y otro tanto (poco más del 14\% de los casos) se encuentran dirigidos contra ambas simultáneamente. Es decir que estas dos grandes empresas de telefonía concentran más de las dos terceras partes de los conflictos del sector (69\% de los casos). En el resto de los conflictos predominan, como parte patronal, las empresas subcontratistas $(21 \%)$ y las cooperativas y otras empresas $(10 \%)$.

Según la base utilizada ${ }^{3}$, cada uno de conflictos responde a una o más causales según la siguiente tipología: i) Conflictos por Condiciones de Trabajo -condiciones de trabajo, salubridad e higiene, flexibilización, jornada, violencia laboral y tipo de contratación-; ii) Conflictos por Causas Salariales -aumentos, adicionales, CCT y paritarias, y jubilatorios-; iii) Conflictos por Representación -prácticas antisindicales, intrasindicales, intersindicales y en el marco de organizar una asociación-; iv) Conflictos por Medidas de Crisis -despidos, despidos masivos, deudas y descuentos, suspensiones y quiebras-; y v) Conflictos por Otras

${ }^{3}$ El presente análisis del conflicto se inicia en el año 2006 dado que es a partir de ese momento que el Observatorio del Derecho Social de la CTA (ODS-CTA) comenzó la construcción de la base de conflictos aquí utilizada. La información oficial elaborado por el Ministerio de Trabajo también se inicia en el año 2006. 
Causales -solidaridad con otros trabajadores, incumplimientos o derogamiento de ley, por puestos de trabajo, en defensa de bienes públicos, etc.-

Recordemos que en el período analizado el sector de las telecomunicaciones se encuentra en una situación de crecimiento de ventas y expansión de los servicios de telefonía móvil e internet. Asimismo en estos años se encuentra vigente un proceso de negociación colectiva que se presenta principalmente bajo la forma de una paritaria anual.

En el siguiente cuadro se sintetizan las múltiples causas de la conflictividad laboral de los 73 casos del panel, para el total del periodo y desagregado por año. Corresponde aclarar que la sumatoria porcentual del total de causas por año supera el $100 \%$ atendiendo que en cada hecho de conflicto se expresan, la mayoría de las veces, más de una motivación.

Cuadro 2. Causas del conflicto laboral en el sector telecomunicaciones, 2006-2015

$\begin{array}{cccccc}\text { Conflictos } & \text { Motivos } & \text { Medidas de } & \text { Condiciones } & \text { Representación } & \text { Otras } \\ & \text { salariales } & \text { Crisis } & \text { de trabajo } & & \text { causas }\end{array}$

\begin{tabular}{l|cccccc}
\hline 2006 & 11 & $36 \%$ & $36 \%$ & $27 \%$ & $9 \%$ & $45 \%$ \\
2007 & 6 & $67 \%$ & $17 \%$ & $33 \%$ & $33 \%$ & $33 \%$ \\
2008 & 0 & $0 \%$ & $0 \%$ & $0 \%$ & $0 \%$ & $0 \%$ \\
2009 & 2 & $100 \%$ & $0 \%$ & $0 \%$ & $50 \%$ & $0 \%$ \\
2010 & 5 & $20 \%$ & $40 \%$ & $40 \%$ & $40 \%$ & $20 \%$ \\
2011 & 12 & $50 \%$ & $50 \%$ & $50 \%$ & $58 \%$ & $25 \%$ \\
2012 & 6 & $17 \%$ & $33 \%$ & $50 \%$ & $17 \%$ & $67 \%$ \\
2013 & 6 & $0 \%$ & $33 \%$ & $83 \%$ & $67 \%$ & $17 \%$ \\
2014 & 5 & $40 \%$ & $20 \%$ & $60 \%$ & $0 \%$ & $60 \%$ \\
2015 & 20 & $10 \%$ & $35 \%$ & $75 \%$ & $10 \%$ & $30 \%$ \\
Totales & $\mathbf{7 3}$ & $\mathbf{3 0 \%}$ & $\mathbf{3 4 \%}$ & $\mathbf{5 3 \%}$ & $\mathbf{2 9 \%}$ & $\mathbf{3 6 \%}$
\end{tabular}

Fuente: Elaboración propia en base a ODS-CTA

Según el cuadro anterior, del total de conflictos del sector se destaca como causa principal la que se refiere a Condiciones de Trabajo (no salariales). Esta causal de la conflictividad está presente en el 53\% de los casos totales del panel, mientras que el resto de las causales se encuentran distribuidas de manera regular en torno al 30\%. Sin embargo como surge de la lectura del Cuadro 2, la conflictividad por condiciones de trabajo no esta homogéneamente distribuida a los largo de todo el período, sino que cobra significatividad 
a partir del año 2011. Hasta ese momento la conflictividad por condiciones de trabajo tenia escasa presencia, en menos de la mitad de los casos, mientras que desde 2011 se encuentra de manera creciente en al menos la mitad de los conflictos de cada año, alcanzando importante niveles en los años 2013 y 2015.

A partir de esta observación podemos dividir el panel en dos etapas basándonos en la identificación de distintas causales que motorizan la dinámica de la combatividad laboral. Así, quedan configurados dos subperíodos: 2006/2010, con 24 casos de conflicto y 2011/2015 con 49 casos. Ambos períodos se diferencian además por distintos niveles de conflictividad, siendo el segundo momento el mas intenso, con un promedio cercano a 10 conflictos por año, muy superior al promedio del primer momento, menor a 5 conflictos por año. Como se observa en el cuadro 3, el motor de la conflictividad en los primeros años ha sido las reivindicaciones salariales con presencia en $46 \%$ de los casos (11 sobre 24), mientras que entre los años 2011 y 2015, la presencia de esta causal en los conflictos cae al $22 \%$. La conflictividad a partir de 2011 se explica fundamentalmente por las motivos de condiciones de trabajo (65\% de los casos vs. $29 \%$ en el primer período).

Cuadro 3. Causas del conflicto en el sector telecomunicaciones por subperíodos

\begin{tabular}{|c|c|c|c|c|c|c|}
\hline & Conflictos & $\begin{array}{l}\text { Motivos } \\
\text { salariales }\end{array}$ & $\begin{array}{l}\text { Medidas } \\
\text { de Crisis }\end{array}$ & $\begin{array}{l}\text { Condiciones } \\
\text { de trabajo }\end{array}$ & Representación & $\begin{array}{l}\text { Otras } \\
\text { causas }\end{array}$ \\
\hline $2006 / 2010$ & 24 & $46 \%$ & $29 \%$ & $29 \%$ & $25 \%$ & $33 \%$ \\
\hline $2011 / 2015$ & 49 & $22 \%$ & $37 \%$ & $65 \%$ & $31 \%$ & $37 \%$ \\
\hline Total & 73 & $30 \%$ & $34 \%$ & $53 \%$ & $29 \%$ & $36 \%$ \\
\hline
\end{tabular}

Fuente: Elaboración propia en base a ODS-CTA

La primera observación que nos surge analizando las causas entre los dos períodos en el conflicto es que la disputa por las condiciones de trabajo se torna mas significativa en el segundo subperíodo, por sobre la disputa salarial. Asimismo observamos que en el segundo período se intensifica también los conflictos por Medidas de Crisis y de Representación, configurando un escenario de mayor complejidad en el conflicto. Nos referimos a complejidad para describir las tensiones entre trabajo y capital que se expresan bajo la forma de despidos y suspensiones, deudas salariales, prácticas antisindicales y tipos de contratación, aún en un marco de ciclo ascendente del sector. De esta manera observamos que ambos subperíodos no solo se encuentran caracterizados por distintos niveles de conflictividad, sino que también se refieren a conflictos cualitativamente distintos.

La conflictividad puede expresarse de diversas formas que se presentan de manera individual o conjunta, es decir que un conflicto puede expresarse a través de más de una medida (paros, marchas, piquetes, etc.). Para todo el período analizado la medida más adoptada por los trabajadores en conflicto ha sido la marcha y/o movilización, presente en 
el $26 \%$ de los casos, seguido por medidas de paro en un $22 \%$ de los conflictos. Las otras medidas recolectadas por la base se refieren a: declaración de Estado de alerta y movilización, Asambleas, Medidas comunicacionales, Trabajo a reglamento, Tomas de establecimientos, Actividades en vía pública, Cortes, Acciones legales y/o administrativas, Medidas de Solidaridad y Piquetes. Para todo el período ninguna de estas otras medidas se encuentra presente en más de11\% de los casos, sin embargo algunas medidas se presentan con mayor frecuencia en el segundo subperíodo identificado (cuadro 4).

Cuadro 4. Principales medidas adoptadas en los conflictos del sector, 2006-2015

\begin{tabular}{|c|c|c|c|c|c|c|c|}
\hline & Conflictos & Paros & Tomas & $\begin{array}{c}\text { Actividades } \\
\text { en vía } \\
\text { pública }\end{array}$ & Cortes & $\begin{array}{l}\text { Movilización } \\
\text { y /o marchas }\end{array}$ & Piquetes \\
\hline $2006 / 2010$ & 24 & $50 \%$ & $8 \%$ & $0 \%$ & $0 \%$ & $21 \%$ & $0 \%$ \\
\hline $2011 / 2015$ & 49 & $8 \%$ & $8 \%$ & $16 \%$ & $10 \%$ & $29 \%$ & $6 \%$ \\
\hline Total & 73 & $22 \%$ & $8 \%$ & $11 \%$ & $4 \%$ & $26 \%$ & $7 \%$ \\
\hline
\end{tabular}

Fuente: Elaboración propia en base a ODS-CTA

Como observamos en el cuadro precedente, mientras que la combatividad de los trabajadores durante los primeros años analizados se expresó principalmente como medidas de paro (y movilización y marchas en menor medida), durante el segundo momento la dispersión de medidas adoptadas en los conflictos resulta más significativa. En esta segunda etapa ya no resulta tan claro identificar una medida vedette que describa la conflictividad general sino que aparece una batería de medidas que responden a las características puntuales de cada conflicto. Recordemos que el primer período se encuentra motorizado por motivos salariales, mientras que en los últimos años la conflictividad se sustenta en reclamos por condiciones de trabajo (ausencia de herramientas de trabajo o elementos de seguridad, flexibilidad, tipos de contratación, etc.). Esta mayor dispersión de las medidas adoptadas se caracteriza además por cierta radicalización de la protesta, atendiendo a un mayor número de conflictos que se expresan mediante medidas como los cortes y piquetes. Son 10 y $6 \%$ respectivamente de los conflictos del subperíodo, versus ausencia de este tipo de medidas en los conflictos del primer momento.

En cuanto a los actores sindicales que participan de estos conflictos cabe destacar que en el primer período se presenta la Foetra Bs. As. motorizando un tercio de los conflictos, seguido por conflictos llevado adelante por trabajadores sin representación gremial (21\% de los casos). En el segundo momento los conflictos son liderados por el Uettel (37\% de los casos) seguido por los conflictos del Cepetel (20\% de los casos). Los conflictos de la Foetra Bs. As. del primer período respondieron a causas salariales y por condiciones de trabajo, y se expresaron mayormente bajo la forma de paro, mientras que los conflictos del Uettel del segundo momento se refieren en su totalidad a conflictos por condiciones de trabajo. Asimismo estos conflictos se yuxtaponen con medidas de crisis, es decir, despidos y 
suspensiones, deudas salariales, cierres de establecimientos, etc. y tomaron la forma Actividades en vía pública, Paros y Marchas.

Como dijimos, la creciente conflictividad del segundo momento se refiere fundamentalmente a luchas por condiciones de trabajo, y en este marco los conflictos poseen un determinante particular. Al observar las diversas demandas que están contenidas en los casos de este tipo, resulta con claridad la emergencia de la tercerización como componente fundamental del conflicto por condiciones de trabajo, y asimismo significativo como variable de explicación de la conflictividad general. Esta observación resulta reforzada por la identificación del Uettel como el sindicato de mayor participación en los conflictos del segundo período. Recordemos que este sindicato ha nacido en 2010 al calor de disputar la efectiva representación de los trabajadores tercerizados de las telecomunicaciones que se encontraban mayormente representados por el sindicato de la construcción por realizar tareas de plantel externo. Pasemos entonces a analizar el componente de tercerización en los conflictos del sector atendiendo a que se presenta como el emergente más significativo en esta segunda etapa.

\section{La tercerización como motor del conflicto laboral}

La tercerización del proceso de trabajo o descentralización productiva es efectivamente una de las principales estrategias desplegadas por las grandes empresas de telecomunicaciones del sector (Montes Cató, 2003; Dávolos, 2009; Ynoub, 2012). Con esta práctica se pretende hacer uso de manera más flexible de la fuerza de trabajo contratada, al tiempo que le permite a la empresa contratista reducir los volúmenes de capital fijo adelantado necesarios para la producción. Lo primero se logra mediante la utilización de distintas cantidades de trabajadores en función del volumen de tareas a realizar en un momento particular, pudiendo incrementarlo o reducirlo en función de la necesidad puntual. De este modo es la empresa contratista la que realiza las tareas para el capital principal y establece con los trabajadores una relación de dependencia que en muchos casos resulta más flexibilizada que la que gozan los empleados de planta de las grandes telefónicas. Así es común advertir que la remuneración percibida por este grupo de trabajadores tercerizados es menor, es variable (en función a la cantidad de servicios realizados) y en ocasiones no se encuentra formalmente registrada ${ }^{4}$. Por el lado del capital fijo, la tercerización permite a la gran empresa de telefonía reducir por ejemplo el volumen de gasto atendiendo a que de este modo puede evitarse la compra y mantenimiento de algunos equipos (que quedan a cargo de la contratista) o reducir la cantidad de edificios y vehículos necesarios para la operatoria. En otros trabajos hemos avanzado en la reconstrucción del proceso productivo total identificando la red de subcontratación y los distintos anillos de contratistas, las tareas externalizadas, etc. (Rodríguez Miglio, 2015a). Asimismo la relación de la tercerización con el conflicto y la estrategia sindical también ha sido abordada por otros autores, enfatizando la posibilidad de articular demandas entre trabajadores de planta y tercerizados (Dávolos, 2009).

\footnotetext{
${ }^{4}$ Esto puede tomar la forma de trabajadores no registrados, trabajadores independientes o cooperativas de
} trabajadores que cuentan con un único cliente a la empresa contratista. 
Si consideramos a la tercerización laboral como una causal en si misma (con independencia del resto de los conflictos por condiciones de trabajo) y retomamos el análisis del total del período, este tipo de conflictos explican el $42 \%$ de los casos del panel analizados (31 casos sobre 73), aunque sin embargo se concentran fundamentalmente en los últimos años. Mientras que en los primeros cinco años la conflictividad por tercerización surge en el $21 \%$ de los casos, a partir de 2011 comienza a emerger como uno de los motivos predominantes en las situaciones de conflicto del sector alcanzando presencia en más de la mitad de la totalidad de los casos del panel (53\%). Este hecho resulta más explícito hacia los años 2013 y 2015 en los cuales la conflictividad por tercerización tiene presencia en 83 y en el $65 \%$ de los casos de cada año, respectivamente.

Grafico 3. Participación de la tercerización como causal de la conflictividad

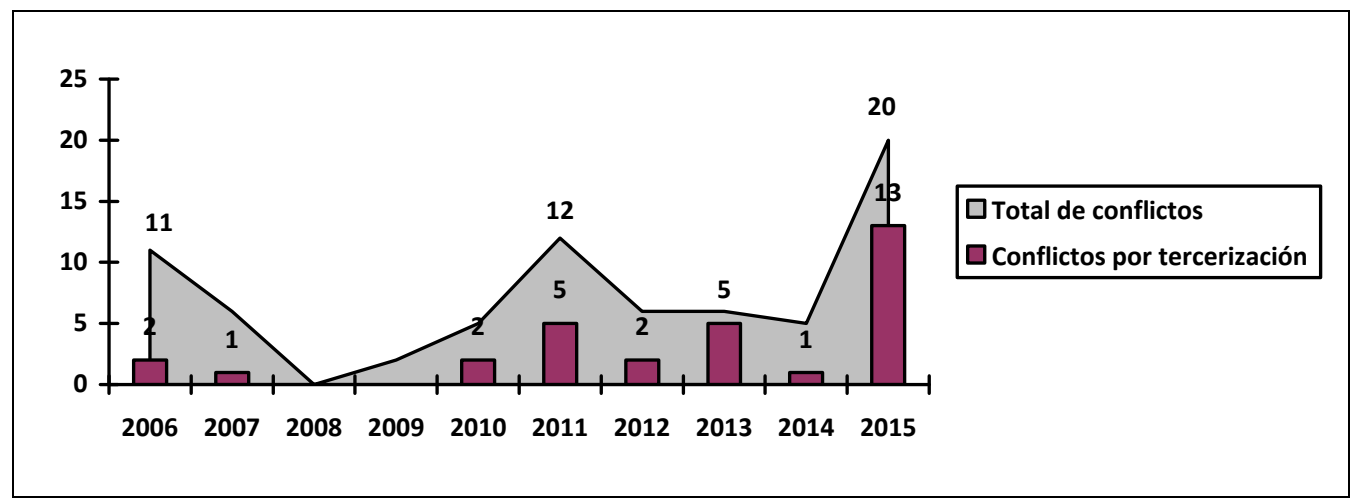

Fuente: Elaboración propia en base a ODS-CTA

Los conflictos por tercerización suelen estar fuertemente asociados con situaciones de crisis en el marco del establecimiento y con los conflictos por representación. Esto se verifica al observar que de la totalidad de los conflictos por tercerización (31 casos para todo el período), un $58 \%$ de los mismos se desarrollan conjuntamente con reclamos ante despidos, suspensiones, quiebras, etc. Asimismo, al igual que en el marco de la conflictividad general del sector, la forma mas adoptada por la protesta se refiere a la marcha/movilización y los paros, seguido por actividades en la vía pública. Sin embargo cobran mayor relevancia otras medidas en comparación con el nivel general, medidas más radicalizadas como tomas de establecimiento, cortes y piquetes.

Cuadro 5. Principales medidas adoptadas en los conflictos por tercerización, 2006-2015

\begin{tabular}{|c|c|c|c|c|c|c|c|}
\hline & Conflictos & Paros & Tomas & $\begin{array}{l}\text { Actividades } \\
\text { en vía } \\
\text { pública }\end{array}$ & Cortes & $\begin{array}{l}\text { Movilización y } \\
\text { /o marchas }\end{array}$ & Piquetes \\
\hline $2006 / 2010$ & 5 & $60 \%$ & $0 \%$ & $0 \%$ & $0 \%$ & $20 \%$ & $0 \%$ \\
\hline $2011 / 2015$ & 26 & $15 \%$ & $15 \%$ & $23 \%$ & $15 \%$ & $23 \%$ & $12 \%$ \\
\hline Total & 31 & $23 \%$ & $13 \%$ & $19 \%$ & $13 \%$ & $23 \%$ & $10 \%$ \\
\hline
\end{tabular}

Fuente: Elaboración propia en base a ODS-CTA 
De los 31 casos identificados, cerca del $61 \%$ de los conflictos por tercerización para los diez años analizados fueron motorizados por la organización gremial Uettel que representa a los trabajadores técnicos tercerizados del sector. Estos conflictos totales fueron dirigidos en un $44 \%$ contra las grandes empresas del sector (6\% contra Telecom, 29\% contra Telefónica y $10 \%$ contra ambas) mientras que el mayor número de conflictos (48\%) se desarrolló en el ámbito de empresas contratistas (Cobra, Plantel, Phono, Redes, Reegam Retesar, etc.). El segundo sindicato con mayor presencia en estos conflictos ha sido el Cepetel en 4 oportunidades, y los conflictos restantes fueron protagonizados por trabajadores sin representación (3), la Foetra Bs. As. (2), Utatec (2), y la Uocra (1).

De los conflictos aquí analizados surgen algunos elementos para pensar la relación entre la emergencia de la protesta, el ciclo económico y la protesta laboral. Los casos de conflicto evidencian con gran claridad los efectos que la descentralización y externalización de la producción tienen sobre los trabajadores telefónicos en el marco de un proceso de crecimiento del sector. En este marco surge una pluralidad de actores y tensiones, no solo entre capital y trabajo, sino también entre las mismas organizaciones gremiales en pugna por ejercer una efectiva representación de los obreros del sector, como expresa la emergencia de un sindicato particular que disputa por la efectiva representación de trabajadores telefónicos que hasta el momento se encontraban encuadrados bajo el convenio de otro sector de actividad. Asimismo surge otro elemento que complejiza la situación, nos referimos a la tensiones entre la doble sujeción patronal que suelen expresarse como un reclamo tanto al empleador formal, como a la telefónica para quien se desempeña la tarea. Sin embargo en muchas ocasiones observamos que el conflicto laboral por tercerización, si bien se opone a la patronal contratista, también tiene como objetivo la interpelación a la telefónica que opera como empresas contratante. Esto es así dado que para los diversos colectivos de trabajadores, la solución al conflicto laboral puede estar bajo la órbita de estas grandes compañías de telecomunicaciones, aún cuando la relación laboral formal se encuentra entablada con una intermediaria.

Por otro lado, el análisis de las reivindicaciones sostenidas por los trabajadores en conflicto nos revela las fuertes diferencias en las condiciones de trabajo de los subcontratados que no se limitan a cuestiones salariales (inferiores, no registrados), sino que además son alcanzados por un costo laboral que debe ser asumido por lo trabajadores siempre que la tarea debe realizarse con elementos propios (herramientas, elementos de seguridad, vehículos, etc.).

Este tipo de situaciones es de vital importancia para entender la dinámica de la conflictividad aquí analizada. El paso de un período de luchas por motivos salariales del primer período hacia conflictos por condiciones de trabajo en el segundo revela que la estrategia de descentralización y externalización de la producción no solo tiene como fundamento cierta optimización de recursos en el seno de las grandes empresas de telecomunicaciones, sino que también opera como una herramienta para la precarización del trabajo y a su vez, para la fragmentación o dispersión de los colectivos de trabajadores de distintas áreas o tareas contenidas en el proceso productivo en cuestión. 


\section{Ciclo económico y protesta laboral. Los antecedentes del debate}

La relación entre desempeño económico y conflictividad laboral no es un tema nuevo en las ciencias sociales. Las fluctuaciones económicas y la evolución de las rebeliones sociales bajo distintas formas ha sido objeto de estudio por un gran número de teóricos de distintas disciplinas. Los aportes fundamentales sobre este tópico podríamos adjudicárselos a Eric Hobsbawm y Ernesto Screpanti, quienes apoyándose en las teoría de las ondas largas de Mandel y los ciclos de Kondratiev, abordaron esta relación.

La dimensión fundamental del debate se refiere al grado de correlación entre el ciclo económico y el desencadenamiento de una rebelión, ya sea popular o estrictamente laboral. Según Hobsbawm (1952) muchos autores han relacionado estas explosiones rebeldes con momentos de auge económico en las que se presentan condiciones favorables para la negociación ${ }^{5}$. Siguiendo esta tradición, más recientemente encontramos un modelo que relaciona ciclos largos e insurrecciones proletarias elaborado por Ernesto Screpanti. Para este autor, las cuatro insurrecciones proletarias más agudas de la historia del capitalismo se corresponden con el momento de auge de cada uno de los ciclos Kondratiev. El fundamento de esta correlación desarrollado por Screpanti es que la tendencia de largo plazo de la tasa de crecimiento depende del estado de ánimo de los capitalistas: cuando la combatividad obrera aumenta, los capitalistas disminuyen el nivel de inversión y se contrae el ciclo de crecimiento. Según este autor, la combatividad de los trabajadores depende de la brecha entre las reivindicaciones declamadas y las efectivamente obtenidas. Así, cuando la tasa de crecimiento permite aumentar el bienestar de los trabajadores, el ciclo económico se desarrolla con bajos niveles de conflictividad. Esto refuerza el estado de ánimo capitalista y aumenta la inversión privada, entonces el ciclo se mantiene en alza. Sin embargo, la mejora sustancial de los trabajadores es acompañada con una mayor frustración producto de la brecha que surge entre lo que desean y lo que obtienen, acumulando así tensiones que explotarán en algún momento, a pesar del auge. De este modo se introducen las expectativas obreras como variable determinante del ciclo de protesta. Ante el cambio de comportamiento que deriva en la explosión de la conflictividad obrera, el ánimo de los capitalistas hace contraer el volumen de inversiones y tira el ciclo para atrás. La crisis hace que, tarde o temprano, las expectativas de los trabajadores se reduzcan, entonces se reducirá su nivel combatividad y mejorará el ánimo capitalista para la reiniciar la inversión. Sin embargo esto puede demorar bastante. Cuando esto sucede, comienza una nueva y larga fase ascendente sin conflicto (Screpanti, 1985).

Hobsbawm rechaza el cumplimiento universal del postulado que asocia auges económicos con conflictividad, fundamentalmente a partir de la evidencia empírica de las explosiones de 1850 en adelante. Explicita que en otros casos el ciclo de protesta aparece como el resultado de una profunda depresión económica que habría infundido en los trabajadores un elevado sentido de la organización (Davis, 1940/41) ${ }^{6}$. A partir de 1860, al

5 Cycles of strikes de A. H. Hansen (1921), British Trade Unions de N. Barou (1947), citados en Hobsbawm, 1952.

${ }^{6}$ The theory of unión growth, de Horace Davis (1940/41), citado en Hobsbawm, 1952. 
menos en Europa, Hobsbawm observa que las explosiones rebeldes se dan de manera generalizada, con independencia del ciclo de auge o crisis, lo cual nos indica que la correlación postulada por Screpanti no sería del todo adecuada.

Hasta mediados del siglo XIX seria acertado suponer que los movimientos sociales resultaron profundamente afectados por unos incrementos catastróficos y simultáneos de la miseria de la población trabajadora. Una posible explicación de esta dinámica puede hallarse en Rostow (1978), donde la tensión social se incrementa a raíz de las depresiones y el consecuente aumento de los precios ${ }^{7}$. Pero a partir de 1850 comienza un proceso en el que varias regiones del mundo se van incorporando a la esfera capitalista, aunque con un grado desarrollo muy heterogéneo, por lo que a nivel general la pauta de las rebeliones ha ido mutando, coexistiendo distintos tipos de procesos. Así, los saltos más llamativos tendieron a producirse cada vez menos en el fondo de las depresiones y cada vez más en los momentos de auge del ciclo, de incremento del empleo o de guerra (Hobsbawm, 1952).

De este modo comienza a tener relevancia la mirada sobre la dinámica de las explosiones en relación con el movimiento de proletarización que se extiende hacia nuevas industrias, nuevas regiones y nuevos actores de la población. Acompañado a esto, comienza a cobrar relevancia el nivel de tecnificación y los cambios en el proceso productivo que podrían impactar intensificando el trabajo y generando condiciones de protesta. Todas estas características complejizan el análisis de modo tal que hallar una pauta que relacione acertadamente el ciclo más general de protesta con el devenir económico resulta cada vez más infructuoso. El propio Hobsbawm describe la heterogeneidad de situaciones: las rebeliones de comienzos de las décadas de 1870 y 1900 surgen en períodos de claras expansiones económicas, pero las de 1830 y de 1880/90 se producen en fases de depresión; la explosión de 1872 se produce a finales de un período de probable descenso del paro y de incremento de los salarios monetarios y reales; la de 1889, durante un período de grave paro, salarios monetarios estables y salarios reales en aumento; la de 1911, en medio de un descenso del paro, estabilidad de los salarios monetarios y descenso de los salarios reales. En los países atrasados se dieron combinaciones. Todo esto sin dejar de observar que la explosividad resultó menor allí donde había instituciones regulares para mantener la negociación de los obreros.

Así las cosas, en el desarrollo de este debate surgen algunas preguntas que merecen ser tenidas en cuenta al momento de abordar el análisis de los procesos de protesta laboral o social y su relación con el desempeño económico. ¿el auge presenta condiciones favorables para la negociación? o ¿la depresión económica infunde un mayor sentido de la organización? La combatitividad obrera ¿depende efectivamente de la brecha entre las expectativas y las reivindicaciones obtenidas? ¿cual es la relación entre la tensión social y la evolución de los precios?

\footnotetext{
${ }^{7}$ En este enfoque una mala cosecha agraria implica el aumento de precios de los alimentos y escasez de materias primas. En un contexto histórico que se caracteriza por la ausencia de política de ingresos y sindicatos muy poco desarrollados, la caída del salario es inevitable. Dado que la mayoría de la población se encontraba en condiciones de ruralidad, el impacto sobre el desempleo es significativo, y el riesgo de amotinamiento sumamente alto.
} 
Volviendo a la relación entre ciclo y conflicto y el análisis antes presentado sobre el sector, el comportamiento de ambas variables hasta 2010 en las telecomunicaciones parece indicar cierta adhesión con la tesis de Ernesto Screpanti en el siguiente sentido: en la medida que el desempeño económico reviste características de auge o crecimiento, el conflicto laboral se incrementa en proporciones similares (ver gráfico 1). Sin embargo la disociación de ambas variables a partir de 2011 nos vuelve a colocar en el terreno de incertidumbre planteado por Eric Hobsbawm. Así las cosas, la mirada general del período nos acerca a la necesidad de analizar las dinámicas concretas, tal como fuera explicitado por el autor inglés, para comprender las características de la protesta. En virtud de estos breves elementos desplegados sobre la historia de la protesta y el análisis realizado a lo largo de la sección, compartimos el reconocimiento por la necesidad del análisis concreto que el mismo Hobsbawm reivindica: "Solo los análisis individuales pueden revelar la combinación especifica de tensiones características de cada una de las explosiones, y es probable que los intentos por descubrir la presencia de una misma combinación estén destinados al fracaso" (Hobsbawm, 1952: 182).

Del análisis pormenorizado del conflicto laboral realizado en este trabajo y su vinculación con la etapa de crecimiento económico permanente a lo largo del período analizado, han surgido algunas reflexiones que compartimos en la siguiente y última sección de este trabajo.

\section{Reflexiones finales}

En el presente trabajo hemos abordado la relación entre ciclo económico y protesta laboral a los fines de profundizar nuestra caracterización sobre la situación imperante en argentina luego de la salida de la crisis de 2001/2002. Así hemos observado a la luz de los movimientos generales de ambas variables en los últimos años, la imposibilidad empírica de adherir con el postulado de Screpanti sobre la conflictividad y el auge económico. Por lo tanto hemos avanzado según la recomendación de Hobsbawm de analizar casos concretos, más específicamente, cualitativos. Esta tarea se acometió para el estudio del sector telecomunicaciones.

En este sector observamos que la trayectoria ascendente del desempeño económico convive con distintas dinámicas del conflicto. En un primer período la expansión del sector se presenta acompañada con un ciclo de protesta en declive, mientras que a partir de 2011, se intensifican los conflictos de la mano de un continuo crecimiento del sector, concluyendo así que la relación entre las variables de conflicto y de desempeño económico en el sector presentan poca o nula correlación, y reforzando el rechazo de la tesis de Screpanti mencionada en el párrafo anterior.

El análisis individual de la conflictividad en el sector nos permitió identificar dos subperíodos con dinámicas de conflicto, no solo cuantitativamente distintas, sino también diferentes en términos cualitativos. Las características principales de ambos subperíodos son resumidas en el siguiente cuadro. 
Cuadro 6. Conflictividad y desempeño económico en las telecomunicaciones argentinas

\begin{tabular}{|c|c|c|c|c|}
\hline Subperíodo & $\begin{array}{l}\text { Ventas } \\
\text { promedio }\end{array}$ & $\begin{array}{c}\text { Evolución } \\
\text { del conflicto }\end{array}$ & Causas destacadas & $\begin{array}{l}\text { Conflictos por } \\
\text { tercerización }\end{array}$ \\
\hline $2006 / 2010$ & 27.186 & $\begin{array}{r}\text { Menor } \\
\text { intensidad }\end{array}$ & Salariales (46\%) & $21 \%$ \\
\hline $2011 / 2015$ & 47.131 & $\begin{array}{r}\text { Mayor } \\
\text { intensidad }\end{array}$ & $\begin{array}{c}\text { Condiciones de trabajo }(65 \%) \\
\text { y Medidas de crisis }(37 \%)\end{array}$ & $53 \%$ \\
\hline
\end{tabular}

Fuente: Elaboración propia en base a CICOMRA, CAC y ODS-CTA

La emergencia de la tercerización como causal de la conflictividad se presenta como uno de los motores más relevantes de este segundo período de la protesta laboral, mas intenso y mas radicalizado en cuanto a las medidas adoptadas, dado que se encuentra presente en más de la mitad de los casos. A partir del análisis cualitativo de este tipo de conflictos se pudieron identificar una serie de elementos que permiten comprender más acabadamente la radicalización de la protesta. Estas características se refieren generalmente a diferencias entre los trabajadores cuya relación laboral se establece con la empresa principal y aquellos trabajadores que, si bien desempeñan tareas para la empresa principal, el empleador es una empresa externa. Las situaciones identificadas se refieren a: i) diferencias salariales, ii) diferencias de encuadramiento o convenio, iii) diferencia en la provisión de los materiales de trabajo y de seguridad, y iv) diferencias entre el representante gremial y la efectiva posibilidad de organización.

Lejos de afirmar que la tercerización en el sector analizado surge como problema laboral en los años recientes, si resulta llamativo que es en estos últimos años un determinante fundamental de la protesta. Esto se ve reforzado con la emergencia de un sindicato como Uettel, que nace al calor de la necesidad de defender las condiciones de trabajo de los telefónicos tercerizados. La emergencia de un sindicato de estas características estaría cristalizando cierta madurez en el sentido de organización en términos de Davis, sin embargo aquí en el marco de una expansión económica, mientras que para el autor la potencialidad de infundir este sentido estaba asociada a las condiciones de depresión económica.

De los conflictos analizados surge con claridad un proceso de radicalización de las medidas adoptadas que es acompañada, quizás a modo de fundamentación de tal comportamiento, por un declive en las condiciones materiales en las que se desempeña la tarea laboral. Este deterioro se expresa como atrasos en las liquidaciones de los salarios, aportes y cargas sociales impagas, ausencia o deterioro de los elementos de seguridad, etc. y se evidencian en la yuxtaposición de las causales de la conflictividad asociadas a Medidas de Crisis, Condiciones de Trabajo y, en menor medida, Causas Salariales.

El ejercicio propuesto en este trabajo, entendemos, aporta a comprender más acabadamente una de las aristas más importantes de las relaciones laborales, a saber, la conflictividad laboral. Pero además, y a partir de la identificación de conflictos que no 
responden principalmente a la disputa salarial, nos permite acercarnos a las implicancias que tienen los cambios en el proceso laboral sobre la clase trabajadora. Este análisis nos ha permitido entender los determinantes de un ciclo de ascenso de la protesta laboral en un sector en auge y con un promedio salarial bien superior a la mayoría del resto de los sectores (solo por debajo de unos pocos sectores como petroleros o algunos informáticos). Sin embargo, a pesar del elevado promedio salarial, la disparidad al interior del sector resulta significativa. No solo está presente la ya conocida diferencia entre trabajadores de empresas grandes versus empresas chicas, sino que en este escenario debe sumarse la diferencia entre trabajadores principales y tercerizados.

De lo desarrollado hasta aquí es poco lo que nos permite referirnos a la relación más general entre ciclo y protesta, pero al menos hemos identificado una vía posible para aportar al problema general, quedando al desnudo ahora la necesidad de extender análisis como el presente, a la mayor cantidad de sectores de la economía argentina como fuera posible. Solo así alcanzaremos una visión general que nos permita ratificar o rechazar las tendencia particulares como las aquí encontradas. Asimismo corresponderá extender temporalmente el análisis aquí desarrollado a los fines de observar la evolución del ciclo de protesta en el sector en la medida que la expansión en términos económicos se retraiga. Esto último será posible siempre y cuando las bases sobre conflictividad laboral continúen elaborándose con el nivel de detalle aquí utilizado.

\section{Bibliografía}

Aruguete, N. y Duarte, M. (2005) "Las estrategias sindicales de los trabajadores telefónicos: las distintas lógicas de acción colectiva" VII Congreso Nacional de Estudios del Trabajo, ASET, Buenos Aires.

Barattini, M. (2013) "La vitalización sindical en el período de la convertibilidad en Argentina” En: Trabajo y Sociedad, Número 20, Santiago del Estero, Argentina.

Cámara Argentina de la Construcción -CAC- (2015) "Análisis del status de los servicios de telecomunicaciones y proyección de la inversión para el período 2016-2025" Recuperado de http://www.camarco.org.ar/biblioteca

Cámara de Informática y Comunicaciones de la República Argentina-CICOMRA(2014) "Informe de mercado de informática y telecomunicaciones" Recuperado de http://www.cicomra.org.ar/cicomra2/asp/estadistica_nota-sb.asp?id_template=6

Dávolos, P. (2009) "Estrategias sindicales frente a la tercerización del trabajo. El caso de las telecomunicaciones en un contexto de crecimiento. (2003-2008). XXVII Congreso de la Asociación Latinoamericana de Sociología. Asociación Latinoamericana de Sociología, Buenos Aires.

Filipetto, S., Pontoni, G. y Trajtemberg, D. (2015) "Mercado de trabajo y relaciones laborales en Argentina entre 2003-2013. Avances y temas pendientes" 12do. Congreso Nacional de Estudios del Trabajo, ASET, Buenos Aires.

Hobsbwam, E. (1952) "Economic fluctuations and some social movements since 1800" En: The Economic History Review, vol. 5, no.1. 
Instituto Nacional de Educación Tecnológica -INET- (2010) "Sector telecomunicaciones. Informe Final" Publicado en

http://catalogo.inet.edu.ar/files/pdfs/info_sectorial/telecomunicaciones-informe sectorial.pdf

Lenguita, P. (2011) Revitalización desde las bases del sindicalismo argentino. En Revista Nueva Sociedad, Nro. 232, marzo-abril. Buenos Aires.

Mandel, E. (1986) "Las ondas largas del desarrollo capitalista. La interpretación marxista" Siglo XXI, Madrid.

Ministerio de Trabajo y Seguridad Social (2015) "Evolución anual de los conflictos laborales" Recuperado de http://trabajo.gob.ar/left/estadisticas/ConLab/index.asp

Ministerio de Trabajo y Seguridad Social (2014) "La disminución de la conflictividad laboral en 2013: menos conflictos, menos huelguistas, menos jornadas no trabajadas". Publicado en http://www.trabajo.gob.ar/left/estadisticas/descargas/conlab/La_ disminuci\%C3\%B3n_de_la_conflictividad\%20laboral_en_2013.pdf

Montes Cató, J. (2003). Disciplina y resistencia en los espacios laborales: en torno a las relaciones de trabajo en las empresas de telecomunicaciones. IV Congreso Latinoamericano de Sociología del Trabajo. La Habana, Cuba.

Observatorio del Derechos Social (ODS-CTA) (2014) "Caída del salario real y crecimiento del conflicto en el sector público”. Informe Anual 2013. Buenos Aires, Marzo. Publicado en http://www.obderechosocial.org.ar/

Observatorio del Derechos Social (ODS-CTA) (2011) "Conflictividad laboral y negociación colectiva". Informe anual 2010. Publicado en http://www.obderechosocial.org.ar/

Rodríguez Miglio, M. (2015) "El proceso (fragmentado) de trabajo en el sector telecomunicaciones" 12do. Congreso Nacional de Estudios del Trabajo, ASET, Buenos Aires.

(2016) "Crecimiento y expansión de las servicios de telecomunicaciones en Argentina durante la posconvertibilidad ¿concentración económica con descentralización productiva?" En: Revista Galega de Economía, Vol. 25-1, Enero-Febrero. Santiago de Compostela, España. Publicado en http://www.usc.es/revistas/index.php/rge

Rostow, W. (1978) "Las etapas del crecimiento. Un manifiesto no comunista" Fondo de Cultura Económica, México.

Screpanti, E. (1985) "Ciclos económicos largos e insurrecciones proletarias recurrentes" En: Zona Abierta, No. 34-35, Madrid.

Senen González, C., Trajtemberg, D. y Medwid, B. (2010) "Tendencias actuales de la afiliación sindical en Argentina: evidencias de una encuesta a empresas" En: Relations industrielles / Industrial Relations, vol. 65, $\mathrm{n}^{\circ}$ 1. Publicado en http://id.erudit.org/iderudit/039526ar

Spaltenberg, R. (2012) "La diversidad de los conflictos laborales: dispersión y centralización en las lógicas de acción de los asalariados privados" Trabajo presentado en VII Jornadas de Sociología de la UNLP. Diciembre, La Plata.

Trajtemberg, D. (2011) "Instituciones laborales y desigualdad salarial: una análisis del efecto de la ampliación de la cobertura de la negociación colectiva entre 2003-2010". 
Martín Rodríguez, Auge económico y conflictividad laboral en las telecomunicaciones argentinas: un análisis de los determinantes de la protesta laboral en el sector durante la posconvertibilidad, Izquierdas, 30, Octubre 2016:288-307

Trabajo presentado en el III Congreso Anual de la Asociación de economía para el desarrollo argentino, AEDA. Agosto, Buenos Aires.

Ynoub, E. (2012). Los sentidos sociales de la subcontratación: organización del trabajo y trabajadores tercerizados por la empresa Telefónica de Argentina. En J. C. Ospina Celis, La subcontratación laboral en América Latina: miradas multidimensionales (pág. 415). Medellín: Escuela Nacional Sindical. 